\title{
Effects of Electric Power Arc Inputs on the Microstructure and Mechanical Properties of $0.4 \% \mathrm{C}$ Steel
}

\section{O. Awheme and Obidimma D. Ikeh}

Department of Mechanical Engineering, Faculty of Engineering, University of Benin, PMB 1154, Benin City, Nigeria.

\section{Article Info}

Received 07 July 2020

Revised 25 July 2020

Accepted 18 August 2020

Available online 31 August 2020

\section{Keywords:}

Microstructure, Welding temperature, Weld metal, Heat affected zone,

Fusion zone, Mechanical properties

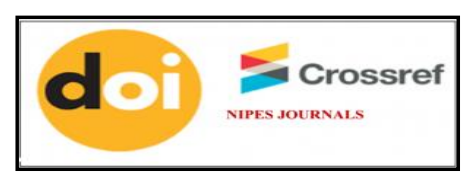

https://doi.org/10.37933/nipes/2.3.2020.33

https://nipesjournals.org.ng (C) 2020 NIPES Pub. All rights reserved

\begin{abstract}
The effect of electric power arc inputs on the microstructure and fracture surface of $0.4 \% C$ steel was analyzed. The power inputs were controlled by varied welding current, arc voltage of 40 volts, welding speed of $3.2 \mathrm{~mm} / \mathrm{sec}$ and electrode gauge of $3.2 \mathrm{~mm}$. The currents were varied at 100A for low heat input, 112.5A for medium heat input and 125A for high heat input. Microstructural and fracture analyses were carried out on the specimens to determine their microstructural configurations. The results showed that increasing the welding current from 100A-125A caused a corresponding increase in microstructure of the specimens. At 100A, the time for solidification was less and resulted in smaller fine grains. At 125A, the time required for solidification increases and yielded coarse grains. On the mechanical properties, the results showed that the selected welding parameters had significant influence on the mechanical properties, the results indicated that the joints made by using low heat input exhibited higher tensile strength (515.90 Mpa) and from scanning electron microscopy (SEM) of tensile test fractured surface exhibited ductile failure, higher hardness strength (179.5 HRC) and impact toughness value of (6.5 Joules) than those welded with medium and high heat inputs respectively which exhibited brittle failure as the heat inputs increases.
\end{abstract}

\section{Introduction}

Medium carbon steels with carbon composition ranging from 0.25 to $0.5 \% \mathrm{C}$, are heat treatable steels and can be successfully welded by all of the arc welding process provided suitable welding procedures and precautions are adopted. The high carbon content of these steels, along with manganese greater than $1 \%$ makes these steels hardenable and weldable, thereby changing phases on cooling to form hard microstructure. For this reason, they are commonly used in the quenched and tempered condition for applications such as production of machine parts like bolts, crankshaft, gears, axles, rail road rails, spanner, hammer heads and heavy-duty forging [1]. Because of the greater likelihood of martensite formation and hydrogen induced cracking during welding, preheating and use of low-hydrogen consumables are necessary to reduce the likelihood of hydrogen induce cracking and high hardness of martensite formed on the parent material [2]. Welding involves the operation of joining two pieces of metal by the application of intense heat, pressure or both to melt the edges of the metals so that they fuse permanently [3]. Welding processes is commonly used in joining sheet metals and, the heat is produced after the electrical energy has been 
converted to light energy which passes through the flux to the electrode to strike an arc, and the light energy is converted to heat energy which helps in welding [4]. The welding process generally involves melting and subsequent cooling and the result of this thermal cycle is distortion, if the welded item is free to move; or residual stresses if the items are securely held or the internal forces still remain [5]. There comes a point when the amount of residual stresses can create potential problems either immediately or during the life of the welded structure and it needs to be reduced or removed, through the process termed stress relieving (tempering) [5]. Also welding, more than any fabrication process exposes the part to rapid and extreme changes that can lead to cracks in the weldment [6]. Usually, the rapid heating and cooling characteristics of welding produces a hard microstructure in the heat affected zone (HAZ), one factor responsible for deterioration and properties of welded joint.

Structure of steel consists of the macro and micro structure. The micro structure is the structure that is visible with the help of the etchant chemical which is poured on the surface of the polished steel [7]. The visible macro-structure is the parent metal, the heat affected zone, the fusion zone [8]. The parent metal is the normal unaffected part of the metal whose structure was not altered. The heat affected zone (HAZ) is the area of the base material of metal that has had its microstructure and properties altered by welding or heat intensive cutting operation. The fusion zone (FZ) is where melting and solidification takes place and the principle controls the size and shape of the grain, segregation and distribution of inclusion and porosity. The microstructure which shows the way the atoms and phases are arranged. This can only be detected by the use of the metallurgical microscope [9]. The internal structure of the plain carbon steel that has been tempered is different from that which has been welded [10]. Mechanical properties of welded joints, the properties related to stress and strain are most often measured to show that such a weld and other similar welds will serve their purpose under loading conditions. Mechanical properties are such properties as strength, hardness and toughness, which not only influence the service life of a component but choice of manufacturing process for that component [11].

Materials are tested for the following reasons [12]: (i) To check chemical composition (ii) To determine suitability of a material for a particular application (iii) To determine data; that is, force deformation (or stress) values to draw up sets of specifications upon which the engineer base his design. (iv) To determine the surface or sub-surface defects in welded materials.

More rarely, several welds are compared to see which welding techniques, process, or chemistries provides the best combination of mechanical properties [2]. Mechanical testing is classified as destructive and non-destructive testing. In destructive testing, the component or specimen either breaks or remain no longer useful for further use and it includes tensile test, hardness test, fatigue test, creep test and impact test.

Tensile tests are carried out to determine the ultimate tensile strength, yield strength and ductility of materials. The test is carried out on a bar of uniform cross section, usually circular (but in some cases flat) in testing machine, which indicates the tensile load being applied. For the very small strain involved in the early part of the test, the elongation of measured length (called the gauge length) is recorded by extensometer [1]. The tensile testing machine is designed to elongate the specimen at a constant rate and to continuously and simultaneously measure the instantaneous applied load and resulting elongations. A stress-strain test typically takes several minutes to perform and is destructive, that is, the test specimen is permanently deformed and usually fractured [13]. Hardness represents the resistance of material to indentation and involves the measurement of plastic deformation caused when a loaded ball or diamond is applied to the surface of the material [1]. Hardness testing of weld provides an indication of two parameters, significant to the determination of successful weld joint and they are: i. Strength ii. Microstructure of a known material. Welding can impose a variety of thermal cycle on steel at various location that produce: $\mathrm{i}$. 
Undesirable hard microstructure susceptible to cracking and brittle fracture ii. Excessively soft microstructure susceptible to plastic collapse under load.

The factors that can influence the resultant hardness includes: pre-heat, weld heat input, cooling rate, total thickness at the weld, alloy content of the steel, alloy content of any flux and the original micro-structural condition of the steel. The hardness can therefore be a useful indicator to determine if the thermal cycle induced by welding has rendered the heat affected zone (HAZ) adjacent to the weld susceptible to cracking or plastic collapse. It has been reported that welding of medium carbon steel is more difficult than welding of low carbon steels because of the greater tendency of martensite formation in the heat affected zone (HAZ) and this makes the weldment susceptible to hydrogen induced cracking and therefore leads to catastrophic failure [11]. Research has shown that medium carbon steel fail in the industry due to poor choice of welding parameters and welding practice and as a result leads to gaseous molecule entrapment, irregular grain sizes and internal stress in weldment and heat affected zone which affects its microstructural and mechanical properties when subjected in service i.e. under load bearing capacity [14]. Therefore, the aim of this study is to investigate the effect of electric power arc inputs on the microstructure and fracture properties on this steel, so that adequate measures can be adopted to improve the fracture and micro-structural properties when subjected in service; under load bearing capacity.

\section{Methodology}

\subsection{Materials}

The materials used for this research work is hot rolled ribbed medium carbon steel rod of $16 \mathrm{~mm}$ diameter and $1 \mathrm{~m}$ long obtained from Universal Steel Rolling Mill, Ogba-Ikeja, Lagos; Nigeria and the chemical composition of the steel analysis was determined at the same company using the mass analyzer (Chemical composition as shown in Table 1). The equipment used for this research work are: mass analyzer, lathe machine, vice, hack saw, Variosfabrieken Groningen shielded metal arc welding machine, a low hydrogen electrode having a rating E6013 and a composition of $0.12 \% \mathrm{C}$, $0.1 \% \mathrm{Si}$ and $0.45 \% \mathrm{Mn}$. The electrode is coated with titanium-potassium materials which can be operated in all positions. It has a diameter of $3.2 \mathrm{~mm}$ and a length of $350 \mathrm{~mm}$ which has an advantage of deep penetration. Others include angle grinding machine, wire brush, file, silicon carbide paper, motor driven polishing machine, etchant chemical, metallurgical microscope with in-built camera, scanning electron microscope.

\subsection{Method}

\subsubsection{Sample preparation}

The $16 \mathrm{~mm}$ ripped medium carbon steels rod was turned (using lathe machines) to $13 \mathrm{~mm}$ diameter and the welding samples were sectioned using a hacksaw into twelve (12) pieces. Three (3) pieces each served as control sample for micro-structural properties and were un-welded and the other nine (9) were welded. The edge that were prepared for the weld geometry is single " $V$ ", groove butt weld each beveled around the edges with the aid of a grinding machine to an angle of $30^{\circ}$ to the horizontal. The beveled faces were cleaned properly and smoothened to ensure sound weld. Heat generated was minimized to avoid changes in the microstructure of the specimens and surface uniformity was ensured when using lathe machine.

\subsubsection{Welding process}

The welding process used is SMAW with E6013- low hydrogen electrode and with the following welding parameters: Welding currents at $100 \mathrm{~A}$, welding current of $112.5 \mathrm{~A}$ and current of $125 \mathrm{~A}$ respectively, welding voltage of $40 \mathrm{~V}$ each, a welding speeds of $2.5 \mathrm{~mm} / \mathrm{sec}$ and electrode diameter of $3.2 \mathrm{~mm}$. The faces of two pieces of the beveled rods were placed $5 \mathrm{~mm}$ apart from each other, and welding machine was appropriately set with proper amperage and voltage. The electrode was placed 
in the holder and the welding machine was turned on. The assembly was tack-welded to ensure alignment and an arc was struck. A single bead was made to ensure uniform fusion of the rods. The weld was de-slagged, cleaned and welded again. The finished bead was spread round the joint to ensure proper weld. After the final welding process, the specimen was allowed to cool on the floor and subsequently a chipping hammer was used to remove the hard slag from the surface of the welds and the specimens were allowed to cool before further investigations were carried out. An analysis of the weldments of medium carbon steel was carried out to determine the micro-structural properties with reference to the parent metal, HAZ, and the weld metal. An alternating current supply was used in filling completely the V-Notch samples which maintains an arc gap of $3 \mathrm{~mm}$ in between. In accordance with this fundamental fact, three different heat input combinations corresponding to different welding currents were selected for this study, i.e. 100A (low heat input), 112.5A (medium heat input), 125A (high heat input). The reasons for using these specific welding current values are two-fold:

i. Firstly; this spectrum of heat input combinations results in arc energies which are sufficient to cause adequate fusion of the base and weld metal selected for the present study

ii. Secondly; a step increases of $12.5 \mathrm{~A}$ was anticipated to be sufficient enough to cause a direct and significant influence on the microstructure and mechanical properties of the welded joint.

The heat inputs were calculated according to Equation (1).

$$
\mathrm{Q}=\left(\frac{V \times I \times 60}{S \times 1000}\right) \times E \text { fficiency }
$$

Where $Q=$ heat input $(\mathrm{KJ} / \mathrm{mm}), V=$ voltage $(\mathrm{v}), I=$ Current $(\mathrm{A})$ and $S=$ welding speed $(\mathrm{mm} / \mathrm{min})$.

The samples were clamped firmly on the vice to prevent movement during welding. The completely filled welded joints were thereafter ground with grinding machine in order to level off and clean the weld with the base metal to standard dimension. During and after welding the joints were visually inspected for their quality and it was ensured that all weld beads possessed good geometrical consistency and were free from visible defects like surface porosity, blow holes.

\subsubsection{Temperature Measurement}

A thermocouple was used to measure the temperature variation across the weld regions. The K-type thermocouple with range of temperature of $-200{ }^{\circ} \mathrm{C}$ to $+1350{ }^{\circ} \mathrm{C}$ was used. The thermocouple was placed at different zones of the weld; immediately after filling the $\mathrm{V}$-notched grove. One at the fusion zone, the others at the heat affected zone and the unaffected base metal and the temperature readings were taken at interval of five seconds until a fairly constant temperature was obtained. Before measuring the temperature of the weld, the initial temperature reading of the thermocouple was noted and recorded.

\subsubsection{Micro-structural Examination}

The micro-structural examination was carried out as follows:

\section{a. Grinding}

Silicon carbide paper was used. Samples for micro-structural examination were ground using a set of abrasive papers rubbed to and fro on the strips starting with the roughest cloth ( 240 grit), until all traces of saw cuts were removed. The specimens were turned through $90^{\circ}$ and rubbed on the next (finer) paper (320 grit) until the previous scratches were removed and then ground on (400 grit) and finally on (600 grit) each time turning through $90^{\circ}$. 


\section{b. Mechanical polishing}

This was done in two stages, with a coarse and a fine abrasive or polishing agent respectively. The specimen was held against horizontal rotating wheel and polished on a rotating disc of a synthetic velvet polishing cloth impregnated with micron alumina paste and finally polished with diamond paste.

\section{c. Etching}

The specimens were then etched with $5 \mathrm{ml}$ nitric acid (Nital) and $95 \mathrm{ml}$ alcohol by submerging or swabbing with this chemical reagent that removes the surface layer produced on polishing and attacks preferentially grain boundaries and second phase precipitates.

\section{c. Microscopic viewing}

The samples were illuminated by a reflected light which was mounted on the microscope. The specimens were viewed with an optical microscope and scanning electron microscope through the eyepiece of the microscope so as to determine the microstructure and fracture surface, which was subsequently recorded by the attached camera to obtain the micrograph. This was viewed at a magnification of X200 and X500.

\subsubsection{Mechanical Properties}

\section{a. Hardness Test}

Hardness values of the specimens were determined using LECO micro-hardness tester with a load of 150 kilos and a dwell time of 10secs. Multiple hardness tests were performed on each sample and the average of the best values taken as a measure of the specimen. The LECO micro-hardness tester calculates the hardness values in Rockwell Hardness Number (HRC). This test was carried out at mechanical engineering department, University of Nigeria Nsukka, Enugu, Enugu State Nigeria. The hardness tests for the specimens were evaluated at three points:

$>$ The weld pool region

$>$ The heat affected zone (HAZ)

$>$ The unaffected base metal.

\section{b. Tensile Test}

The tensile tests were conducted on the samples at room temperature using Instron Electromagnetic Testing Machine of ISO 6892 standard at the load of 200KN. The ends of the specimens were gripped in the machine and load was applied until failure occurred. The tensile test specimen has the following dimensions: Length $=100 \mathrm{~mm}$, Gauge length $=80 \mathrm{~mm}$, Diameter $=6 \mathrm{~mm}$, Width of the grip section $=10 \mathrm{~mm}$, radius $=10 \mathrm{~mm}$. The results are presented in Table $4.5-4.9$ and Figure 4.2

\section{c. Impact Test}

The impact tests were conducted on the samples at room temperatures using impact testing machine at the department of mechanical engineering workshop, University of Benin. V-notch bevel was made on each of the test samples using an angle grinding machine. The samples were placed horizontally as a simply supported beam between two anvils $40 \mathrm{~mm}$ apart in such a way that the striking hammer strikes the specimen on the face which is opposite to the notched. The striking hammer or pendulum was made to fall freely. As the pendulum hits the samples, it destroyed the samples from V-notch.

\section{Results and Discussion}

Table. 1. Chemical composition of the steel.

\begin{tabular}{|l|l|l|l|l|l|l|l|l|l|l|l|l|l|l|l|l|}
\hline Elements & $\mathrm{C}$ & $\mathrm{Si}$ & $\mathrm{Mn}$ & $\mathrm{S}$ & $\mathrm{P}$ & $\mathrm{Cr}$ & $\mathrm{Cu}$ & $\mathrm{Ni}$ & $\mathrm{Nb}$ & $\mathrm{Al}$ & $\mathrm{B}$ & $\mathrm{W}$ & $\mathrm{Mo}$ & $\mathrm{V}$ & $\mathrm{Ti}$ & $\mathrm{Fe}$ \\
\hline Composition & 0.4 & 0.3 & 0.9 & 0.1 & 0.1 & 0.2 & 0.3 & 0.1 & 0.01 & 0.01 & 0.01 & 0.01 & 0.01 & 0.01 & 0.01 & 97.6 \\
\hline
\end{tabular}


O. Awheme and Obidimma D. Ikeh / NIPES Journal of Science and Technology Research 2(3) 2020 pp. 350-359

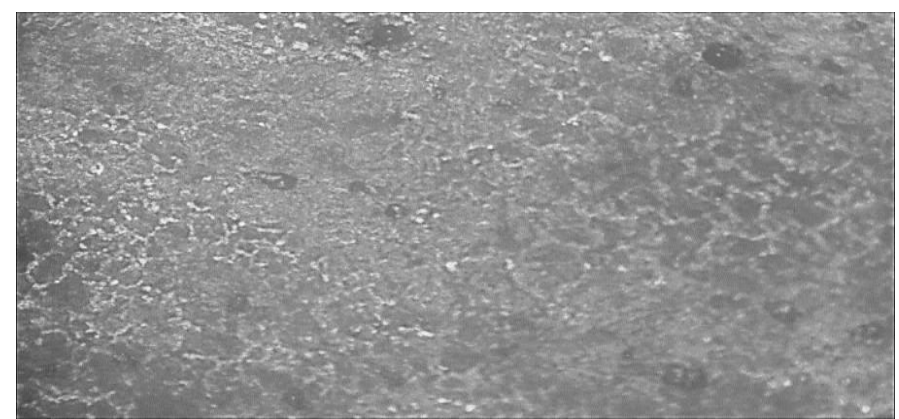

Plate 1. Micrograph of Control Steel Specimen at 200X

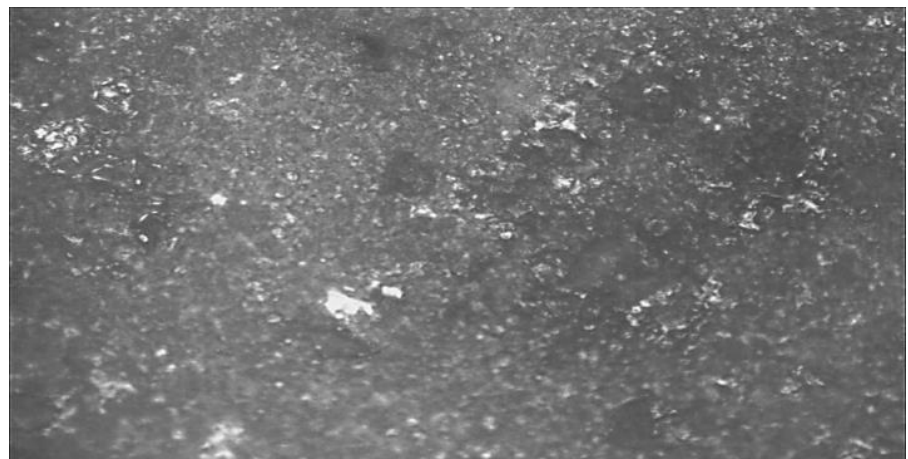

Plate 2. Micrograph of Test Specimen for Low Heat Input (100A) at 200X

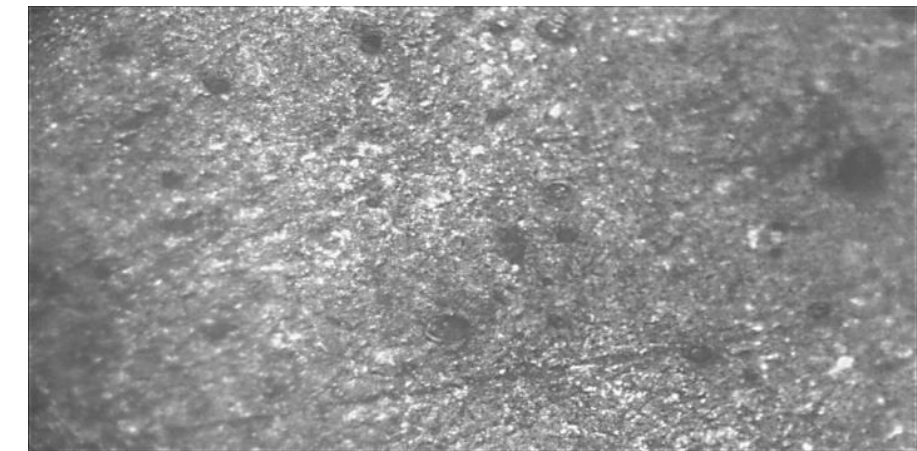

Plate 3. Micrograph of Test Specimen for Medium Heat Input (112.5A) at 200X

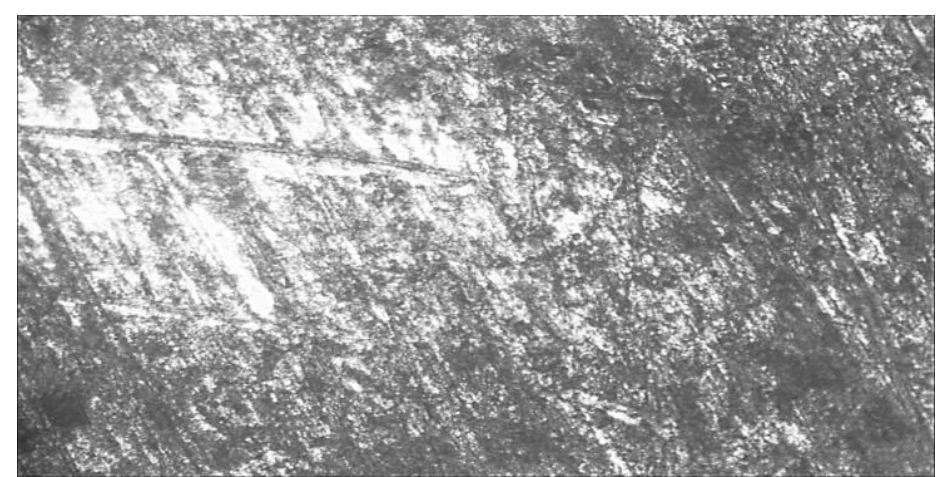

Plate 4. Micrograph of Test Specimen for High Heat Input (125A) at 200X 
O. Awheme and Obidimma D. Ikeh / NIPES Journal of Science and Technology Research 2(3) 2020 pp. 350-359

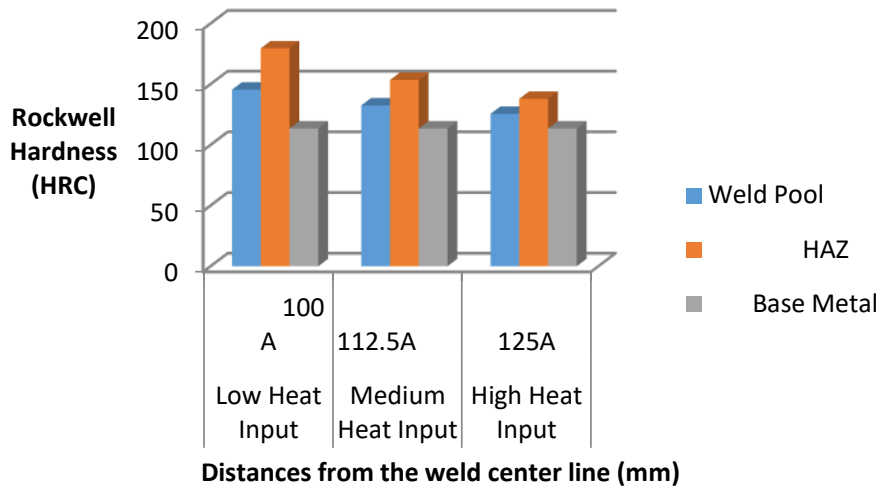

Figure 1. Micro-hardness Results at different zones of the weldments with different heat inputs

The hardness test result shows that increase in heat inputs; results in decrease in hardness value

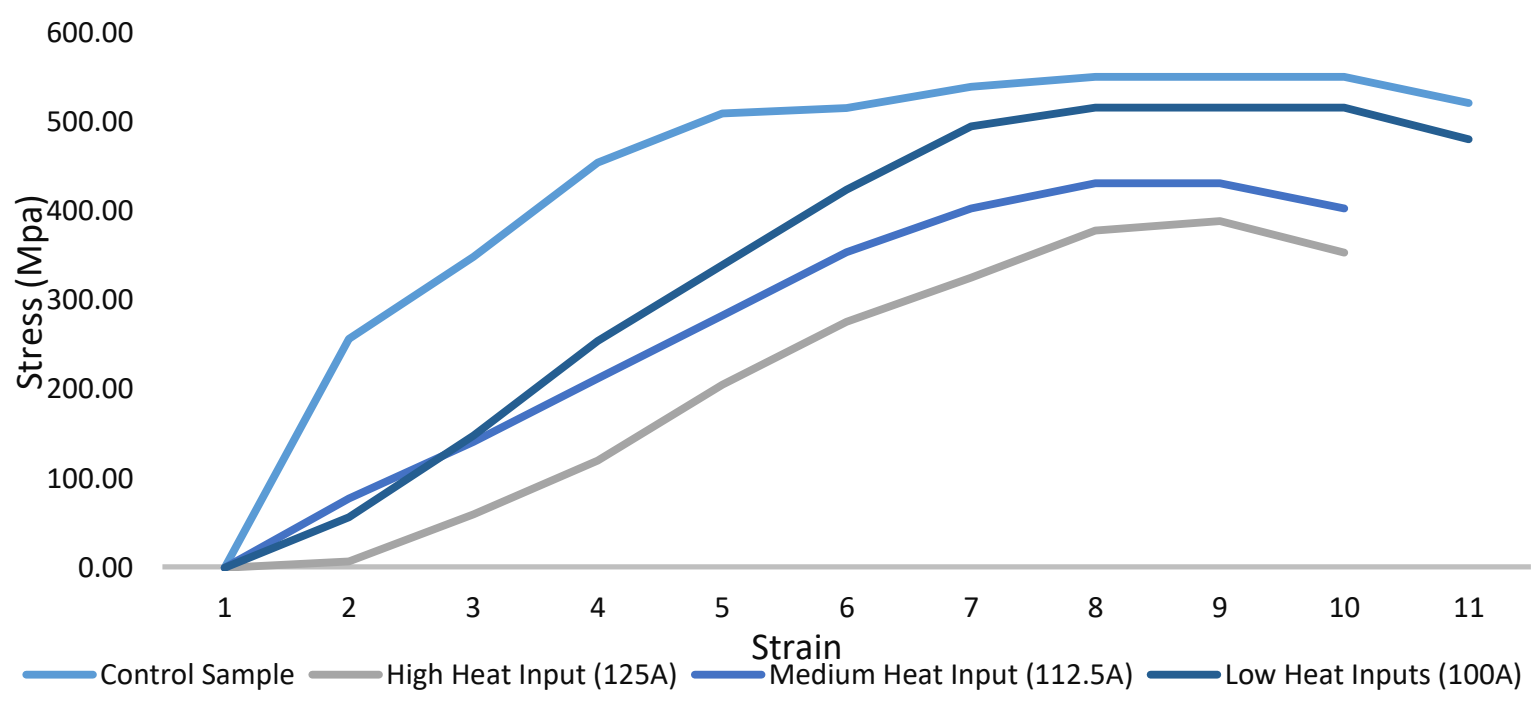

Figure 2. Tensile Test Results of the Samples

The higher the heat inputs, the lower the tensile strength, i.e. the tensile strengths are proportionally decreasing with an increase in heat input.

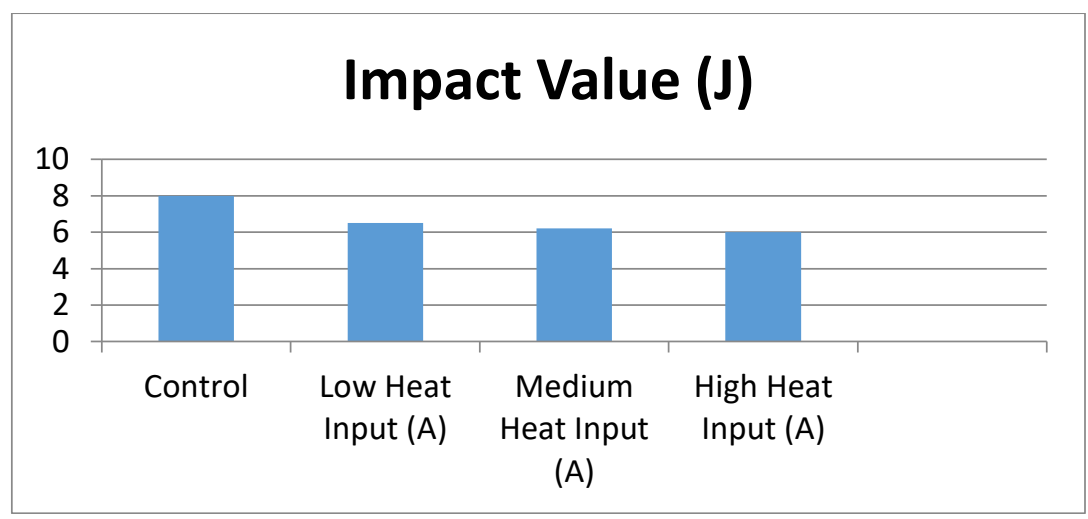

Figure 3. Impact Test Results at Different Heat Inputs 
The higher the heat inputs, the lower the impact toughness value, i.e. the impact toughness values are proportionally decreasing with an increase in heat input.

The microstructures of the medium carbon steel were illustrated clearly in Plate 1-4, for the structures of as-received and as welded samples. The microstructures contain some colonies of pearlite which is represented by brown region; ferrite is represented by white region and cementite \{iron carbide $\left.\left(\mathrm{Fe}_{3} \mathrm{C}\right)\right\}$ represents the black region in Plate 1-4. The microstructure of as-received steel in Plate1. contains fine ferrite and pearlite, while microstructure of as-welded samples varies across the weldment; and it was observed that a large amount of pearlite is present in the ferrite matrix. As depicted in Plate 2-4, as the current was increased, the pearlite become finely distributed within the coarse ferrite matrix with an increase in the proportion of the ferrite in pearlite, and with ranges of fine dispersion of iron carbide in a strained ferrite matrix in the weld and in the HAZ [7]. The weld microstructure is controlled mainly by the cooling cycle. At lower energy input (i.e. with low level of current) the time for solidification is less. This rapid cooling promotes smaller grains that leads austenite to transform into martensite and form fine grain microstructure and as compared with higher energy input, the time required for solidification decreases and therefore cooling rate slows down which makes austenite to have enough time to transform to pearlite and yields coarse grain microstructure [4]. This Coarse grain in the microstructure indicates lower hardness, tensile and impact strength. This is in agreement with [7] who observed that this cooling rate which is a function of the heat input utilized determined the proportion of ferrite and pearlite formation in the microstructure of the weldments and that these in turn, influences the tensile, impact and hardness behavior of weldments. Conclusively, it can be stated low heat input produces fine microstructure and in turn improves the mechanical properties of the welded samples. However, increasing the heat input values causes' expansion towards the microstructure's grain sizes; which becomes coarse and decreases the mechanical properties which led to the loss of ductility at the welded joint, and resulted in brittleness of the material [14].

The transverse tensile strength of all the welded specimen made using different heat inputs conditions has been evaluated. In each condition, three specimens were tested and the tensile strength of the three specimens per unit heat inputs was obtained. The tensile results so obtained shows that maximum tensile strength of $179.5 \mathrm{Mpa}$ is possessed by the specimen made using low heat input combination followed by 153.5 Mpa using medium heat input and 137.9 Mpa using high heat input combination. The tensile strength of the weldment was observed to be lower compared to as-received sample, this is as a result of thermal stresses stored in the weldment during the welding operation and it is in agreement with [13] who observed that the high tensile strength and good ductility is possessed by the joint at low heat input, which can be attributed to smaller dendrite sizes and lesser inter-dendritic spacing in the fusion zone. Relatively lower tensile strength and ductility is possessed by the joints with long dendrite sizes and large inter-dendritic spacing in the fusion zone of the joint welded using higher heat inputs. This is supported by [9] who noted that with increased current, the heat generated increased causing the grains to recrystallized and grow in size. Increase in grain size decreases the tensile strength whereas decrease in grain size increases. the tensile strength. Figure 2 show that the tensile strengths of medium carbon steel used decreases with increase in welding parameters (welding current, voltage, and welding speed). It is shown that during low heat input condition, the grain size of the HAZ is small which varies when changes to higher heat input. During higher heat input, it was observed that carbide precipitates a lot along the grain boundaries leading to sensitized zone around grain boundaries which helps in grain coarsening of HAZ zone and significant grain coarsening was observed in the HAZ of all the joints and it was found that the extent of grain coarsening in the HAZ zone increased with increase in heat input. This is in agreement with [7] who observed that this behavior was attributed to the fact that increased 
current meant an increase in heat input which could create room for defect formation, thus the observed reduced mechanical properties and they also established that service failure of arc welded joints is due to cracking in the HAZ and also that, the performance of the welded structure is usually limited by failure initiation within the HAZ of the base material, particularly within the coarse-grain region of HAZ adjacent to the weld metal as a result of increase in heat input.

The ability of a material to withstand an applied load is referred to as toughness. The transverse impact strength of all the welded specimen made using different heat inputs conditions has been evaluated. In these conditions, three specimens with control specimen were tested and the impact strength of the three specimens with the control specimen per unit heat inputs was obtained. It was observed that the impact toughness values are proportionally decreasing when heat inputs increase. This is in agreement with [4] who observed that this attribute is as a result of fine ferrite and pearlite matrixes distributions in low heat input when compared with coarse ferrite and pearlite matrixes distributions as heat input increases. From Figure 3, it was observed that the control specimen has the maximum impact toughness value of 8 Joules $(\mathrm{J})$, followed by the low heat input with impact toughness value of 6.5 Joules $(\mathrm{J})$. At medium heat input and high heat input, the impact toughness values are 6.2Joules $(\mathrm{J})$ and 6Joules $(\mathrm{J})$ respectively. Therefore, low heat input has the best impact strength of 6.5 Joules $(\mathrm{J})$ while the high heat input has the lowest impact strength of 6 Joules $(\mathrm{J})$. These results showed that the increase in heat input coarsens the grain structure both in the weld metal and heat affected zone and is in agreement with [9] who noted that the notch toughness of the coarse grained HAZ decreases with an increase in energy input. They also found that stress relieving reduced the notch toughness of both the weld metal and HAZ as a result of embrittlement caused by carbide precipitation

\section{Conclusion}

The analysis of microstructures of the welded specimens confirmed the heat input directly affected the mechanical properties and microstructure of the weldment. In general, the higher the heat inputs, the slower the cooling rate which resulted in coarse grains in both HAZ and weld metal while the lower the heat input, the fast the cooling rate which resulted in fine microstructure. On the mechanical properties, the results showed that the selected welding parameters had significant influence on the mechanical properties, the results indicated that the joints made by using low heat input exhibited higher tensile strength (515.90 Mpa) and from scanning electron microscopy (SEM) of tensile test fractured surface exhibited ductile failure, higher hardness strength (179.5 HRC) and impact toughness value of (6.5 Joules) than those welded with medium and high heat inputs respectively which exhibited brittle failure as the heat inputs increases.

\section{References}

[1] Onyekpe, B.O. (2002). The Essentials of Metallurgy and Materials in Engineering: Nigeria, Ambik Press Benin City, Pp. 6-143, ISBN. 978-8016-53-7.

[2] ASM International (1991). Metals Handbook, Vol.8-Mechanical Testing and Evaluations: USA, Pp. 97-105. ISBN. 80-7306-185-3

[3] Parma, R.S. (2010). Welding Engineering and Technology: 2nd Edition: New Delhi, Khanna Publishers, Pp. 18-353.

[4] Mohammed. R.A, Abdulwahab M and Dauda E.T. (2013). Properties Evaluation of Shielded Metal Arc Welded Medium Carbon Steel Material. International Journal of Innovative Research in Science, Engineering and Technology, Vol. 2, No. 8, Pp. 3351-3357.

[5] Welding Technology Institute of Australia (2006). Hardness Testing of Welds, Pp. 1-2.

[6] Desia, P. (2010). Monitoring Heat Treatment to Improve Weld Quality: Welding Journal-The American Welder, Vol 3, No 2, Pp. 109-111. 
O. Awheme and Obidimma D. Ikeh / NIPES Journal of Science and Technology Research 2(3) 2020 pp. 350-359

[7] Asibeluo I.S and Emifoniye E. (2015). Effect of Arc Welding Current on the Mechanical Properties of A36 Carbon Steel Weld Joints. SSRG International Journal of Mechanical Engineering, Vol. 2, No. 9, ISSN. 2348-8360. Pp. 32-40.

[8] Rajput, R.K (1999). Material Science and Engineering, 3rd Ed. NaiSarak, Delhi-110006: S.K Kataria and Sons Publishers, ISBN. 81-85749-92-2.

[9] Callister, W.D. (2007). Materials Science and Engineering-An Introduction: USA, John Wiley \& Sons, Inc., Pp. 365-367.

[10] Abioye T.E (2017). The Effect of Heat Input on the Mechanical and Corrosion Properties of AISI 304 Electric Arc Weldments. British Journal of Applied Science and Technology. Vol20, No.5, ISSN. 2231-0843. Pp. 1-10.

[11] Roger Timings (2008). Fabrication and Welding Engineering, 1st Ed. MA01803, USA: Newness Publishers, ISBN. 978-7506-6691-6.

[12] Choudary. R.B. (2007). Materials Science and Metallurgy, $1^{\text {st }}$ Ed. NaiSarak, Delhi-110006: Khanna Publisher.

[13] Dodo.M. R, Ause. T, and Adamu. M.A (2016). Effect of Post-Weld Heat Treatment on the Microstructure and Mechanical Properties of Arc Welded Medium Carbon Steel. Nigerian Journal of Technology,35(2), pp. 337343.

[14] Wan S.H, Nurul, S.M and Sarizam, M. (2015). Effect of Welding Heat Input on Microstructure and Mechanical Properties at Coarse Grain Heat Affected Zone of ABS Grade A Steel. APRN Journal of Engineering and Applied Science, 10(20), pp. 9487-9495. 